\title{
Terrorist Networks, Network Energy and Node Removal: A New Measure of Centrality Based on Laplacian Energy
}

\author{
Xingqin $\mathbf{Q i}^{1,4}$, Robert D. Duval ${ }^{2}$, Kyle Christensen ${ }^{3}$, \\ Edgar Fuller ${ }^{4}$, Arian Spahiu ${ }^{2}$, Qin $\mathrm{Wu}^{5}$, Yezhou $\mathrm{Wu}^{4}$, \\ Wenliang Tang ${ }^{4}$, Cunquan Zhang ${ }^{*}$ \\ ${ }^{1}$ School of Mathematics and Statistics Shandong University (Weihai), Weihai, China \\ ${ }^{2}$ Department of Political Science West Virginia University, Morgantown, USA \\ ${ }^{3}$ Department of Political Science Columbus State University, Columbus, USA \\ ${ }^{4}$ Department of Mathematics West Virginia University, Morgantown, USA \\ ${ }^{5}$ School of IOT Engineering Jiangnan University, Wuxi, China \\ Email: *cqzhang@math.wvu.edu
}

Received November 8, 2012; revised November 11, 2012; accepted January 9, 2013

\begin{abstract}
In this work we propose a centrality measure for networks, which we refer to as Laplacian centrality, that provides a general framework for the centrality of a vertex based on the idea that the importance (or centrality) of a vertex is related to the ability of the network to respond to the deactivation or removal of that vertex from the network. In particular, the Laplacian centrality of a vertex is defined as the relative drop of Laplacian energy caused by the deactivation of this vertex. The Laplacian energy of network $G$ with $n$ vertices is defined as $E_{L}(G)=\sum_{i=1}^{n} \lambda_{i}^{2}$, where $\lambda_{i}$ is the eigenvalue of the Laplacian matrix of $G$. Other dynamics-based measures such as that of Masuda and Kori and PageRank compute the importance of a node by analyzing the way paths pass through a node while our measure captures this information as well as the way these paths are "redistributed" when the node is deleted. The validity and robustness of this new measure are illustrated on two different terrorist social network data sets and 84 networks in James Moody's Add Health in-school friendship nomination data, and is compared with other standard centrality measures.
\end{abstract}

Keywords: Network; Centrality; Laplacian Energy; 9/11 Hijacking; Bali Bombing; Terrorism

\section{Introduction}

The recent growth in the use of Social Network Analysis (SNA) to understand complex networks demonstrates the importance of the implicit connections within groups that arise from day to day social activity. Similarly, our increasing interest in observing, detecting and analyzing terrorist networks leads us to be extremely interested in understanding who is central to the functionality of these groups that form around the common goal of engaging in terrorist activities. As a result, researchers and analysts from several areas have a strong interest in understanding centrality within networks for both academic and operational reasons.

SNA provides us tools for mapping and measuring relationships and flows between people, groups, organizations, computers, URLs, and many other connected bits of information/knowledge. The vertices in political networks, of which terrorist networks have emerged as one of the most salient, are typically people, organizations, or

\footnotetext{
*Corresponding author.
}

groups while the edges show relationships, connections, or flows between the vertices. SNA provides both a visual and a mathematical analysis of these interrelationships. Recent studies of networks in political science range from such diverse topics as international conflict [1], terrorism [2] and policy networks [3] to disciplinary introspection about job placement in political science [4].

To understand network structure and the entities being studied, we often start with an evaluation of their location relative to all other actors in the network. For networks, the most readily examined measure of location means how close is the object to the center, or centrality. The finding of some important vertices with high centralities in order to characterize the properties of the networks has significant uses in many fields. These include synchronization transition, the spread of epidemics, and the transmission of information. For example, in diffusive systems the vertices with large degree play a crucial role, which are decisive in resolving the traffic jam at a bottleneck [5].

The ability to measure centrality in social networks 
has been a particularly useful development. These ideas have materialized in many well known centrality measures such as degree centrality, along with closeness, betweenness, eigenvector, subgraph centrality, Katz prestige and Bonacich centrality, etc. These measures are certainly not the only measures of centrality, and it is clear that these measures capture different aspects of the positioning of the nodes [6]. Given how varied networks can be, it is not surprising that there are many different ways of viewing position, centrality or power in a network. But note that these standard methods also have their own weakness. For example, as have been stated, "The simplicity of degree method is an advantage and also is an disadvantage: only the local structure around a vertex is calculated and it does not take into consideration the global structure of the network; for example, although a vertex might be connected to many others, it might not be in a position to reach others quickly to access resources, such as information or knowledge [7]”; "Betweenness method considers the global network structure and also can be applied to networks with disconnected components, but it is not without limitations; for example, vertices in a network that generally do not lie on a shortest path between any two other vertices will receive the same score of zero [7]". Katz prestige and Bonacich centrality both depend on the choice of parameters heavily. Besides, these existing measures describe either the local environment around a vertex (e.g., degree centrality) or the global position of a vertex in the network (e.g., closeness, betweenness and subgraph centrality). For example, from its definition, "subgraph centrality" tends to find the center (s) of an entire network when in fact we are really interested in finding the center for each community within the network. If the network consists of more than two communities with dramatically different sizes, the nodes in the smaller community would exhibit lower "subgraph centrality" ranks than the ones in larger community, so that the leader in smaller community will not rank highly overall. Thus, an intermediate (between local and global) characterization of the vertex centrality has been claimed as a necessity for the study of, for example, the food web in $[8,9]$ if species to community relations are to be understood. An intermediate centrality approach is also suggested to be the most appropriate if the relative importance of vertex is to be quantified in social networks.

In this paper, based in part on the basic idea of spectral graph theory, we present a novel centrality method that takes into account the Laplacian energy of the graph, a quantity introduced in [10] which reflects the graph's internal connectivity. In particular, the Laplacian centrality of a vertex is defined as the relative drop of Laplacian energy in the network caused by the deactivation of this vertex from the network. Theorem 1 makes precise the way in which the Laplacian centrality of a vertex can be used to reveal its importance in a network. This result says that the Laplacian centrality of a vertex not only takes the local environment around it into account but also the larger environment around its neighbors, making it an intermediate between the global and local characterizations of the position of a vertex in a network. We investigate the validity and robustness of this new measure by illustrating this method on three social network data sets of strong theoretical and substantial policy interest. Examining two terrorists networks (those involving the Bali Nightclub bombing in 2002 [11] and the 9/11 hijackers network [12]) and all the 84 networks in the Add Health in-school friendship nomination data from James Moody, we compare the results of our Laplacian centrality measure to other standard centrality measures and show its reliability.

This paper is organized as follows. We first give some notations and terminology in Section 2. In Section 3, we introduce the new measure-Laplacian centrality, whose simple calculation is presented in Section 4 based on some structural studies of graph theory. Analytical and numerical results on two different networks will be shown in Section 5, where the rankings of vertices for different centrality measures are presented. By comparing with outputs of other methods, the effectiveness of Laplacian centrality method is supported by both known facts (intelligence information) and statistical analysis (consensus comparison), see both Section 5 and Section 6. The computational complexity of various centrality methods are further compared and discussed in Section 7, which will show that Laplacian method has the lower time complexity making it quite advantageous for large scale networks. Conclusions will be reported in Section 8.

\section{Graph Theory Notation and Terminology}

In most contexts, a social network can be effectively represented by a graph where the vertices are the individuals, and the edges represent the social links (connection). In this paper, we consider the symmetric case where social networks are represented by undirected graphs.

Let $G$ be an undirected graph, consisting of a set of $n$ vertices $V(G)=\left\{v_{1}, v_{2}, \cdots, v_{n}\right\}$ and a set of $m$ edges. The number of edges that are incident to a vertex is called the degree of the vertex. Let

$$
A(G)=\left(a_{i, j}\right)_{n \times n}
$$

be the adjacency matrix of the graph $G$, where the element $a_{i, j}$ equals 1 if there is an edge between vertices $i$ and $j$, and 0 if there is not. 


\section{Laplacian Centrality}

In the following, we will first introduce the definition of Laplacian matrix and Laplacian energy for a graph, then define the Laplacian centrality for a vertex.

Let $\mathrm{G}$ be a simple graph (without graph loops or multiple edges) of $n$ vertices, and

$$
D(G)=\operatorname{diag}\left(d_{1}, d_{2}, \cdots, d_{n}\right)=\left(\begin{array}{cccc}
d_{1} & 0 & \cdots & 0 \\
0 & d_{2} & \cdots & 0 \\
\cdot & \cdot & \cdot & \cdot \\
0 & 0 & \cdots & d_{n}
\end{array}\right)
$$

be the diagonal matrix with the vertex degrees

$d_{1}, d_{2}, \cdots, d_{n}$ of its vertices $v_{1}, v_{2}, \cdots, v_{n}$. Define $L(G)=D(G)-A(G)$ as the Laplacian matrix of the graph $G$ (note that the adjacency matrix $A(G)$ is defined in Section 2).

The following is a short list of some properties about the Laplacian matrix $L(G)$ [13].

\section{Properties}

1) $L(G)$ is symmetric, singular and positive semidefinite.

2) All eigenvalues $\lambda_{i}$ are real and nonnegative.

3) The smallest eigenvalue $\lambda_{n}=0$.

4) The multiplicity of zero eigenvalues equals the number of connected components of $G$.

Definition 1 If $G$ is a graph of $n$ vertices, and $\lambda_{1}, \lambda_{2}, \cdots, \lambda_{n}$ are the eigenvalues of its Laplacian matrix. The Laplacian energy of $G$ is defined as the following invariant:

$$
E_{L}(G)=\sum_{i=1}^{n} \lambda_{i}^{2} .
$$

Lemma 1 [10] For any graph $G$ on $n$ vertices with vertex degrees $d_{1}, d_{2}, \cdots, d_{n}$, we have

$$
E_{L}(G)=\sum_{i=1}^{n}\left(d_{i}^{2}+d_{i}\right) .
$$

Lemma 2 [10] If $H$ is an arbitrary subgraph of a graph $G$, then $E_{L}(H) \leq E_{L}(G)$.

We will see from the above lemmas Laplacian energy reflects the internal connectivity of a network. Thus, the relative drop of Laplacian energy in the network caused by the deactivation of this vertex from the network can be easily regarded as a "signal" to show the importance of the removing vertex in the whole network.

Definition 2 If $G$ is a graph on $n$ vertices $\left\{v_{1}, v_{2}, \cdots, v_{n}\right\}$, let $H$ be the graph obtained by removing vertex $v_{i}$ from $G$. The Laplacian centrality $C_{i}^{L}$ of vertex $v_{i}$ is defined as

$$
C_{i}^{L}=(\Delta E)_{i}=E_{L}(G)-E_{L}(H)
$$

Obviously, by Lemma 2, $E_{L}(G)-E_{L}(H)$ must be nonnegative.

\section{Calculation of Laplacian Centrality, a Graph Theory Result}

\subsection{Graph Theoretical Descriptions}

Theorem 1 If $G$ is a graph of $n$ vertices, then the Laplacian centrality with respect to $v$ is

$$
C_{v}^{L}=(\Delta E)_{v}=d_{G}^{2}(v)+d_{G}(v)+2 \sum_{v_{i} \in N(v)} d_{G}\left(v_{i}\right),
$$

where $N(v)$ is the set of neighbors of $v$ in $G$ and $d_{G}\left(v_{i}\right)$ is the degree of $v_{i}$ in $G$.

Proof. Assume that the vertex set of $G$ is $v_{1}, v_{2}, \cdots, v_{n}$, and there are $m$ edges in $G$. It is well known that $\sum_{i=1}^{n} d_{G}\left(v_{i}\right)=2 m$. Then

$$
E_{L}(G)=\sum_{i=1}^{n}\left(d_{G}^{2}\left(v_{i}\right)+d_{G}\left(v_{i}\right)\right)=2 m+\sum_{i=1}^{n} d_{G}^{2}\left(v_{i}\right) .
$$

Without loss of generality, assume $H=G-\left\{v_{1}\right\}$. Note that there are $(n-1)$ vertices $\left\{v_{2}, \cdots, v_{n}\right\}$ and $m-d_{G}\left(v_{1}\right)$ edges in $H$.

$$
\begin{aligned}
E_{L}(H) & =\sum_{i=2}^{n}\left(d_{H}^{2}\left(v_{i}\right)+d_{H}\left(v_{i}\right)\right) \\
& =2\left(m-d_{G}\left(v_{1}\right)\right)+\sum_{i=2}^{n} d_{H}^{2}\left(v_{i}\right)
\end{aligned}
$$

Let $N\left(v_{1}\right)$ be the neighborhood of vertex $v_{1}$ in $G$, the following is obvious:

$$
d_{H}\left(v_{i}\right)= \begin{cases}0, & \text { if } i=1 ; \\ d_{G}\left(v_{i}\right)-1, & \text { if } v_{i} \in N\left(v_{1}\right) ; \\ d_{G}\left(v_{i}\right), & \text { otherwise. }\end{cases}
$$

So

$$
\begin{aligned}
& E_{L}(G) \\
& =2 m+\sum_{i=1}^{n} d_{G}^{2}\left(v_{i}\right) \\
& =2 m+d_{G}^{2}\left(v_{1}\right)+\sum_{i=2}^{n} d_{G}^{2}\left(v_{i}\right) \\
& =2 m+d_{G}^{2}\left(v_{1}\right)+\sum_{v_{i} \in N\left(v_{1}\right)} d_{G}^{2}\left(v_{i}\right)+\sum_{v_{i} \notin N\left(v_{1}\right)} d_{G}^{2}\left(v_{i}\right)
\end{aligned}
$$

and

$$
\begin{aligned}
& E_{L}(H) \\
& =2\left(m-d_{G}\left(v_{1}\right)\right)+\sum_{i=2}^{n} d_{H}^{2}\left(v_{i}\right) \\
& =2\left(m-d_{G}\left(v_{1}\right)\right)+\sum_{v_{i} \in N\left(v_{1}\right)} d_{H}^{2}\left(v_{i}\right)+\sum_{v_{i} \notin N\left(v_{1}\right)} d_{H}^{2}\left(v_{i}\right) \\
& =2\left(m-d_{G}\left(v_{1}\right)\right)+\sum_{v_{i} \in N\left(v_{1}\right)}\left(d_{G}\left(v_{i}\right)-1\right)^{2}+\sum_{v_{i} \notin N\left(v_{1}\right)} d_{G}^{2}\left(v_{i}\right)
\end{aligned}
$$
is 


$$
\begin{aligned}
& (\Delta E)_{1} \\
& =E_{L}(G)-E_{L}(H) \\
& =d_{G}^{2}\left(v_{1}\right)+2 d_{G}\left(v_{1}\right)+\sum_{v_{i} \in N\left(v_{1}\right)}\left[d_{G}^{2}\left(v_{i}\right)-\left(d_{G}\left(v_{i}\right)-1\right)^{2}\right] \\
& =d_{G}^{2}\left(v_{1}\right)+2 d_{G}\left(v_{1}\right)+\sum_{v_{i} \in N\left(v_{1}\right)}\left[2 d_{G}\left(v_{i}\right)-1\right] \\
& =d_{G}^{2}\left(v_{1}\right)+2 d_{G}\left(v_{1}\right)-d_{G}\left(v_{1}\right)+\sum_{v_{i} \in N\left(v_{1}\right)} 2 d_{G}\left(v_{i}\right) \\
& =d_{G}^{2}\left(v_{1}\right)+d_{G}\left(v_{1}\right)+2 \sum_{v_{i} \in N\left(v_{1}\right)} d_{G}\left(v_{i}\right)
\end{aligned}
$$

From Theorem 1, we notice the following facts:

First, the Laplacian centrality agrees with the standard measures on assignment of extremes. For example, it gives the maximum value to the central vertex of a star, and equal value to the vertices of a cycle or a complete graph.

Second, it is well known that the degree centrality of $v$ only considers the information contained in the number of vertices which can be reachable from $v$ directly. The Laplacian centrality of a vertex involves the information of vertices that can be reachable to $v$ within two steps and as a result the Laplacian centrality of a vertex takes not only the local environment around it into account but also the larger immediate environment around its neighbors. It is thus an intermediate measure between global and local characterizations of the position of a vertex within networks. Because of this we should anticipate that it will reveal differences in network structure that emerge out of significant local influence upon areas of the graph. Intuitively, this means that we should expect the Laplacian centrality to yield a more useful vertex characterization when the overall graph structure has subgroups that contribute disproportionately to the overall goals of the network. Hence we should see an increase in the relative importance of actors whose removal from the network would do the most damage to the graph structure. In the case of monitoring terrorist groups, Laplacian centrality may offer significant operational counterintelligence benefits due to its strong association with vertex inactivation. These expectations have been verified by the experimental results in Section 5 .

\subsection{Comparison with Local and Global Centrality Methods}

In this section, we will give two simple examples to show the differences between Laplacian methods with the popular existing centrality measures respectively. Here, degree method and subgraph method are chose to represent local and global standard method respectively.

Please see Figure 1 for the first example. Based on degree centrality, $u$ has higher ranking than $v$ because the degree of $u$ is 4 while the degree of $v$ is 3 .

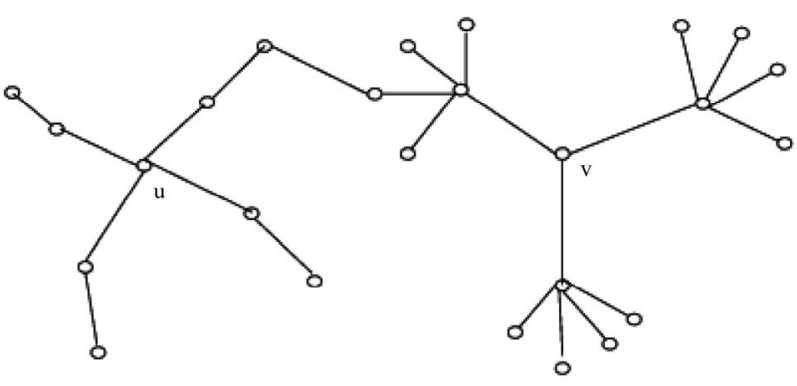

Figure 1. Example 1 for comparison of degree centrality and Laplacian centrality method.

But based on Laplacian method, $v$ would have higher ranking than $u$ because

$$
\begin{aligned}
& (\Delta E)_{u}=d_{G}^{2}(u)+d_{G}(u)+2 \sum_{v_{i} \in N(u)} d_{G}\left(v_{i}\right)=36 \\
& (\Delta E)_{v}=d_{G}^{2}(v)+d_{G}(v)+2 \sum_{v_{i} \in N(v)} d_{G}\left(v_{i}\right)=42
\end{aligned}
$$

Please see Figure 2 for the second example. Centrality method based on global characterization of network tends to find the center(s) of whole network. But at the most time what we are really interested is to find the center for each community in the network. If the network is consist of more than two communities and with dramatically different sizes, the nodes in smaller community would get lower ranks than the ones in larger community, so that the leader in smaller community will not come up with high rank. We specify this fact by the example in Figure 2. The network is consisting of two communities with centers 60 and 10 respectively. From the following table, we will see that based on Laplacian method, we always can find the two communities' centers (\# 10 and \# 60), but with subgraph centrality method, all nodes in the left bigger community all get higher ranks than nodes in right smaller community, which will regard node \# 11 as the second center of the network wrongly.

\begin{tabular}{ccc}
\hline node\# & Laplacian method & subgraph method \\
\hline $\mathbf{6 0}$ & $1 \mathrm{st}$ & $1 \mathrm{st}$ \\
$\mathbf{1 0}$ & 2 nd & $51 \mathrm{st}$ \\
$\mathbf{1 1}$ & 3rd & 2nd \\
\hline
\end{tabular}

Here we only present particular examples which show that sometimes local or global centrality methods can not give the reliable results. Thus in practice, we need intermediate method often.

\section{Applications and Experimental Results}

To demonstrate the effectiveness of Laplacian centrality, we will test it on two terrorist networks: the Jemaah Islamiyah Network collected by Stuart Koschade [11], 


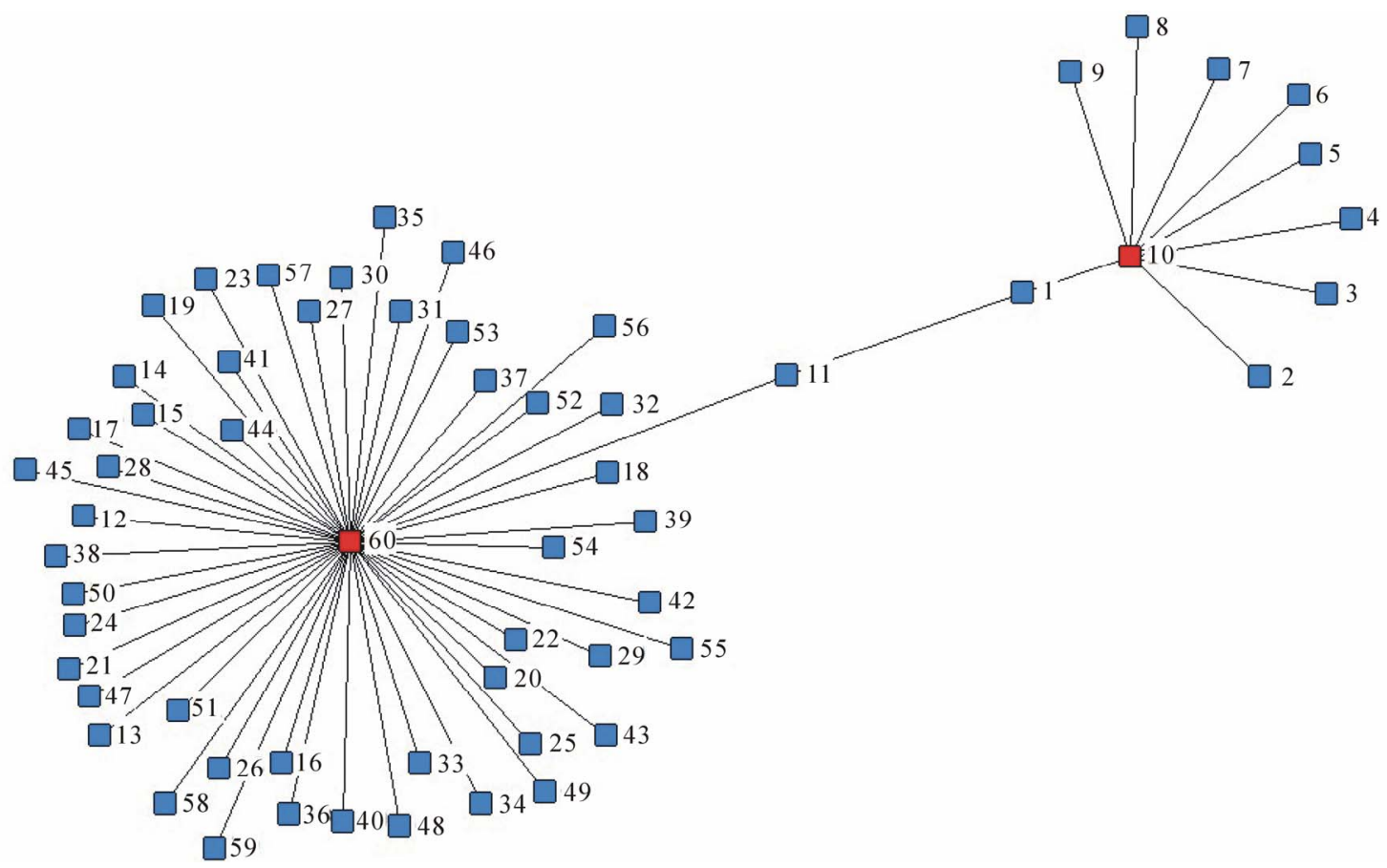

Figure 2. Example 2 for comparison of subgraph centrality and Laplacian centrality method.

and the 9/11 hijacker network assembled by Valdis Krebs [12], and all the 84 networks in the Add Health in-school friendship nomination data. We compare the Laplacian results with the most classical methods including degree, closeness, eigenvector and betweenness centrality methods except Katz prestige and Bonacich centrality methods, because the latter two all depend on the parameters users choice heavily, which means different choice of parameter would affect the results significantly.

\subsection{Data set 1: Jemaah Islamiyah Graph-Bali 2002 Terrorist Attack}

The Jemaah Islamiyah network is comprised of 17 members of the group that participated in the nightclub bombing on December 2002, Bali, Indonesia. The network is a good example of a tight network with two clear clusters (cliques) and a significant actor with strong measures of betweenness and closeness. This terrorist attack was also studied intensively in [14].

\subsubsection{Output Analysis Based on Known Evidence}

The information provided by the four standard centrality measures and our Laplacian centrality measure as applied to the 17 members of the terrorist group directly involved in the operation are provided in Table 1, where the centrality scores are normalized (dividing by the highest score of each method). We also list the rank for each terrorist. Note that frequently actors will exhibit the same scores for a number of measures. In the case when ties occur, we usually assign them the same rank. For example, Sarijo, Imron, Dulmatin, Azahari, Patek and Ghoni all get the same Laplacian score 0.5778, thus we will rank all of them No. 3 since there are only two scores 1.0000 (Samudra) and 0.6222 (Idris) greater than 0.5778 .

The terrorist Samudra, in fact, provides the only link between the bomb makers and Team Lima, the group setting off the bombs (see Figure 3), and not surprisingly scores rather high based on Laplacian and all other four standard centrality methods. Idris, in his role as logistics commander, yielded a high centrality score also, although it is not as significant as that of Samudra. He is ranked as the second most central actor in the network based on Laplacian, degree, closeness and betweenness centrality methods, but is ranked 8 th on eigenvector centrality. And we also find that the members in the bomb construction team (for example, Sarijo, Imron, Dulmatin, etc.) also get high scores based on Laplacian centrality, which support Koschade's conclusion that "the members of the Palau Manjangan residence (bomb construction team) seemingly the center of the operation [11]". We also find that Mubarok is given the lowest rank on Laplacian method, which is consistent with the conclusion in [11] that "the lowest centrality scores were reserved for the contingency members who were kept to the periphery except when called upon for assistance. Mubarok had the lowest scores as he was kept very isolated and did not play any significant part in this stage of the operation.” Lastly, the two actual suicide bombers, Feri and Arnasan, have their highest combined rank us- 
ing Laplacian centrality.

To see the different rankings among the five centrality

Table 1. The scores and ranks based on five centrality methods for the 17 actors in Jemaah Islamiyah's network

\begin{tabular}{|c|c|c|c|c|c|c|c|c|c|c|c|}
\hline \multirow{2}{*}{ Names } & \multicolumn{5}{|c|}{ Scores } & \multicolumn{6}{|c|}{ Ranks } \\
\hline & Laplacian & Degree & Betweenness & Closeness & Eigenvector & Laplacian & Degree & Betweenness & Closeness & Eigenvector & Consensus \\
\hline Samudra & 1.0000 & 1.0000 & 1.0000 & 1.0000 & 1.0000 & 1 & 1 & 1 & 1 & 1 & 1 \\
\hline Idris & 0.6222 & 0.6667 & 0.1008 & 0.7727 & 0.8917 & 2 & 2 & 2 & 2 & 8 & 2 \\
\hline Imron & 0.5778 & 0.6000 & 0.0381 & 0.7391 & 0.8946 & 3 & 3 & 3 & 3 & 2 & 2 \\
\hline Sarijo & 0.5778 & 0.6000 & 0.0273 & 0.7391 & 0.8946 & 3 & 3 & 4 & 3 & 2 & 2 \\
\hline Dulmatin & 0.5778 & 0.6000 & 0.0273 & 0.7391 & 0.8946 & 3 & 3 & 4 & 3 & 2 & 2 \\
\hline Azahari & 0.5778 & 0.6000 & 0.0273 & 0.7391 & 0.8946 & 3 & 3 & 4 & 3 & 2 & 2 \\
\hline Patek & 0.5778 & 0.6000 & 0.0273 & 0.7391 & 0.8946 & 3 & 3 & 4 & 3 & 2 & 2 \\
\hline Ghoni & 0.5778 & 0.6000 & 0.0273 & 0.7391 & 0.8946 & 3 & 3 & 4 & 3 & 2 & 2 \\
\hline Muklas & 0.5689 & 0.6000 & 0.0273 & 0.7391 & 0.8661 & 9 & 3 & 4 & 3 & 9 & 9 \\
\hline Junaedi & 0.2222 & 0.3333 & 0.0000 & 0.6071 & 0.2108 & 11 & 11 & 11 & 10 & 13 & 10 \\
\hline Hidayat & 0.2222 & 0.3333 & 0.0000 & 0.6071 & 0.2108 & 11 & 11 & 11 & 10 & 13 & 10 \\
\hline Octavia & 0.2222 & 0.3333 & 0.0000 & 0.6071 & 0.2108 & 11 & 11 & 11 & 10 & 13 & 10 \\
\hline Rauf & 0.2222 & 0.3333 & 0.0000 & 0.6071 & 0.2108 & 11 & 11 & 11 & 10 & 13 & 10 \\
\hline Arnasan & 0.2222 & 0.3333 & 0.0000 & 0.6071 & 0.2108 & 11 & 11 & 11 & 10 & 13 & 10 \\
\hline Feri & 0.3333 & 0.4000 & 0.0000 & 0.5152 & 0.6125 & 10 & 10 & 11 & 17 & 10 & 15 \\
\hline Amrozi & 0.2089 & 0.2667 & 0.0055 & 0.5862 & 0.3447 & 16 & 16 & 10 & 15 & 11 & 16 \\
\hline \multirow[t]{3}{*}{ Mubarok } & 0.1556 & 0.2000 & 0.0000 & 0.5667 & 0.2536 & 17 & 17 & 11 & 16 & 12 & 17 \\
\hline & & & & & & Laplacian & Degree & Betweenness & Closeness & Eigenvector & \\
\hline & & & & & diviation & 36 & 72 & 139 & 48 & 156 & \\
\hline
\end{tabular}

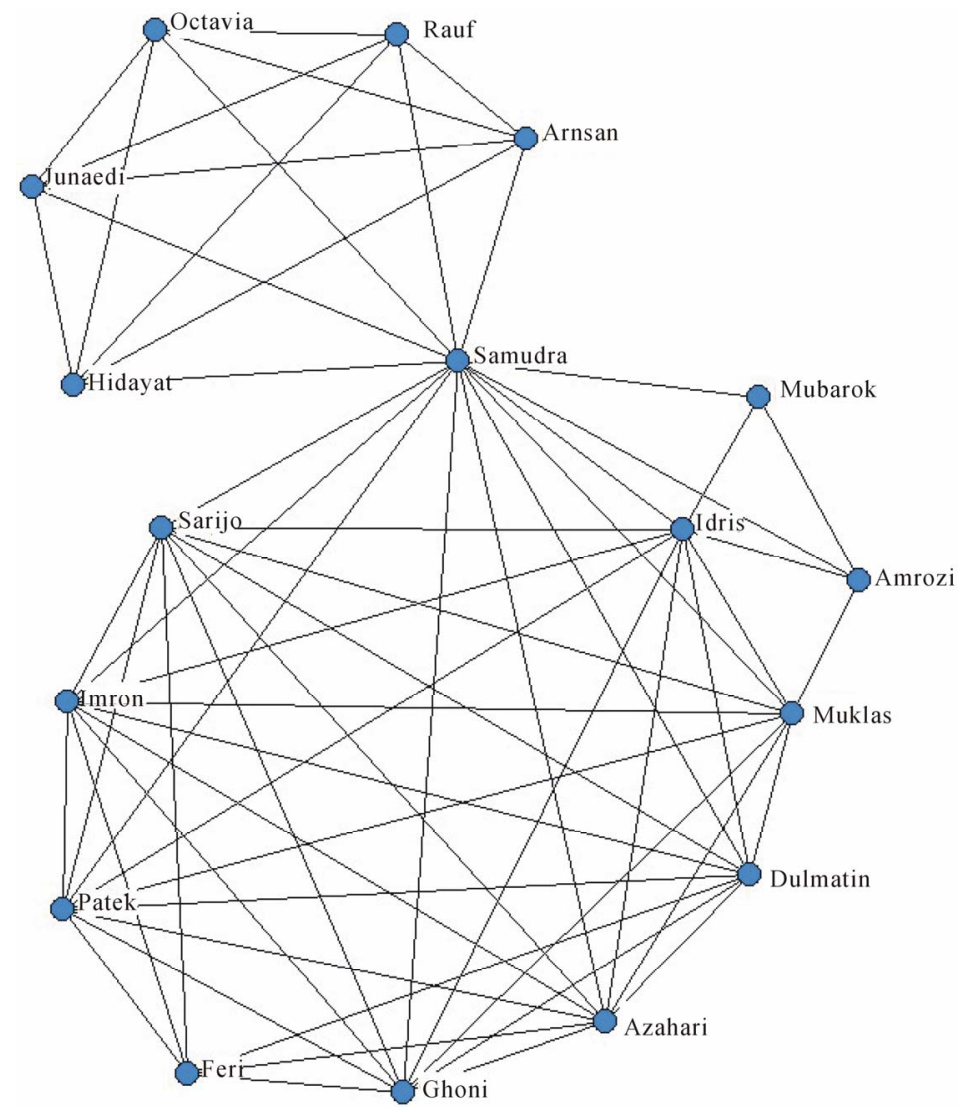

Figure 3. Stuart Koschade's (2006) data on Jemaah Islamiyah's attack on the Bali nightclub in 2002. 
measurements visually, we plot the scores for each method in Figure 4. Ranks obtained by all centrality measures generally agree with each other for this data set. Some noticeable minor disagreements are the ranks of terrorists Muklas and Feri. However, the ranks by Laplacian centrality are consistent with the majority of other measurements.

\subsection{Data Set 2: Valdis Krebs' (2002) Data on the 9/11 Hijackers}

Another widely discussed example of network analysis of terrorist activity is Krebs' now classic analysis of the 9/11 hijackers [12]. Through public data, the network centered around the 19 hijackers of these events were examined by Krebs [12]. We use the larger network (Figure 4 in [12]) which contains numerous additional individuals involved in the support network behind the 19 hijackers who actually conducted the suicide mission. These co-conspirators were conduits for money, communications routes, and provided needed skills and knowledge. Figure 5 shows the hijackers and their network neighborhood - their direct and indirect associates.

The ranking of actors based on four standard centrality measures and Laplacian centrality measure are provided in Table 2, where we only list the centrality scores for these 19 hijackers. The values are normalized (dividing by the highest score of each method). We also rank these 19 hijackers according to their scores and when ties happen we use the same criterion as used in the Jemaah Islamiyah network.

\subsubsection{Output Analysis Based on Known Evidence (Team Leaders of Hijacking Groups).}

There were four commercial airplanes hijacked in this

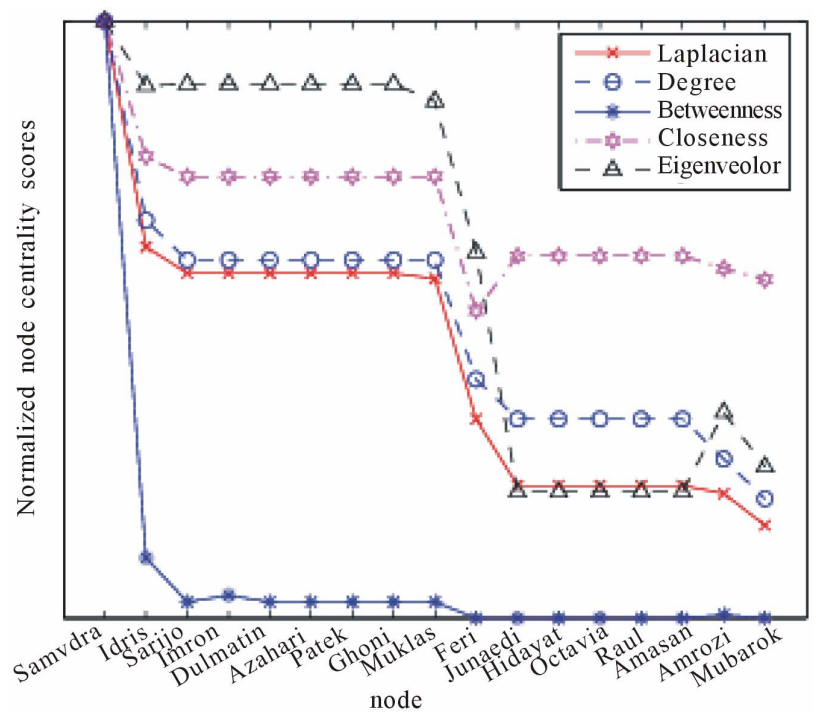

Figure 4. Plots of normalized scores for 17 actors in Bali Operation under five centrality measurements. terror plot. The following is the list of all hijackers on these airplanes.

American Airlines (AA11): Mohamed Atta (pilot), Abdulaziz al-Omari, Satam al-Suqami, Wail al-Shehri, Waleed al-Shehri.

American Airlines (AA77): Hani Hanjour (pilot), Nawaf al-Hazmi, Salem al-Hazmi, Khalid al-Mihdhar, Majed Moqed.

United Airlines (UA175): Marwan al-Shehhi (pilot), Fayez Ahmed, Hamza al-Ghamdi, Ahmed al-Ghamdi, Mohand al-Shehri.

United Airlines (UA93): Ziad Jarrah (pilot), Ahmed al-Haznawi, Saeed al-Ghamdi, Ahmed al-Nami.

We can see from Table 2, Mohamed Atta (AA11) is unanimously identified as the most important actor by all measures. This result is consistent with the findings of The 9/11 Commission Report [15] and verified by statements of Osama bin Laden.

However, beyond that, we observe a significant difference between the Laplacian measure and the other traditional measures (degree, betweenness, closeness and eigenvector centrality). While Mohamed Atta (AA11), Marwan Al-Shehhi (UA175), and Hani Hanjour (AA77) are also the top three in the traditional centrality scores, the increased prominence of Ziad Jarrah (UA93) in Laplacian centrality is striking and noteworthy. Based on Laplacian centrality, the four most important centers comprise all four pilots of the different flights. However, Ziad Jarrah (UA93) scores as either the 5th (degree centrality), 6th (betweenness), or 9th (closeness) most important actor in traditional measures. Eigenvector centrality also places Ziad Jarrah in the top 4, but does so at considerable cost to the assessment of Nawaf Alhazmi. Alhazmi is in the top 5 of the other four centrality measures, and is second in betweenness, indicating his importance as a communications conduit. Eigenvector centrality moves him to 11th place, perhaps too great a reduction for an individual who also trained as a pilot, and met several times with Atta in the planning of the attack. The Laplacian results are somewhat more intuitively appealing.

The normalized values of the five measures provided in Figure 6 shows more variation in various measures, relative to the Laplacian measure. In fact, certain individuals, such as, Ziad Jarrah (UA93) and Nawaf AlHazmi (AA77) shift substantially from index to index. Unlike the Bali data, these are noticeable shifts in the relative importance of various members of the terrorist network.

\subsection{The Add Health in-School Friendship Nomination Data}

The third data set we use here is the Add Health inschool friendship nomination data, which are constructed 


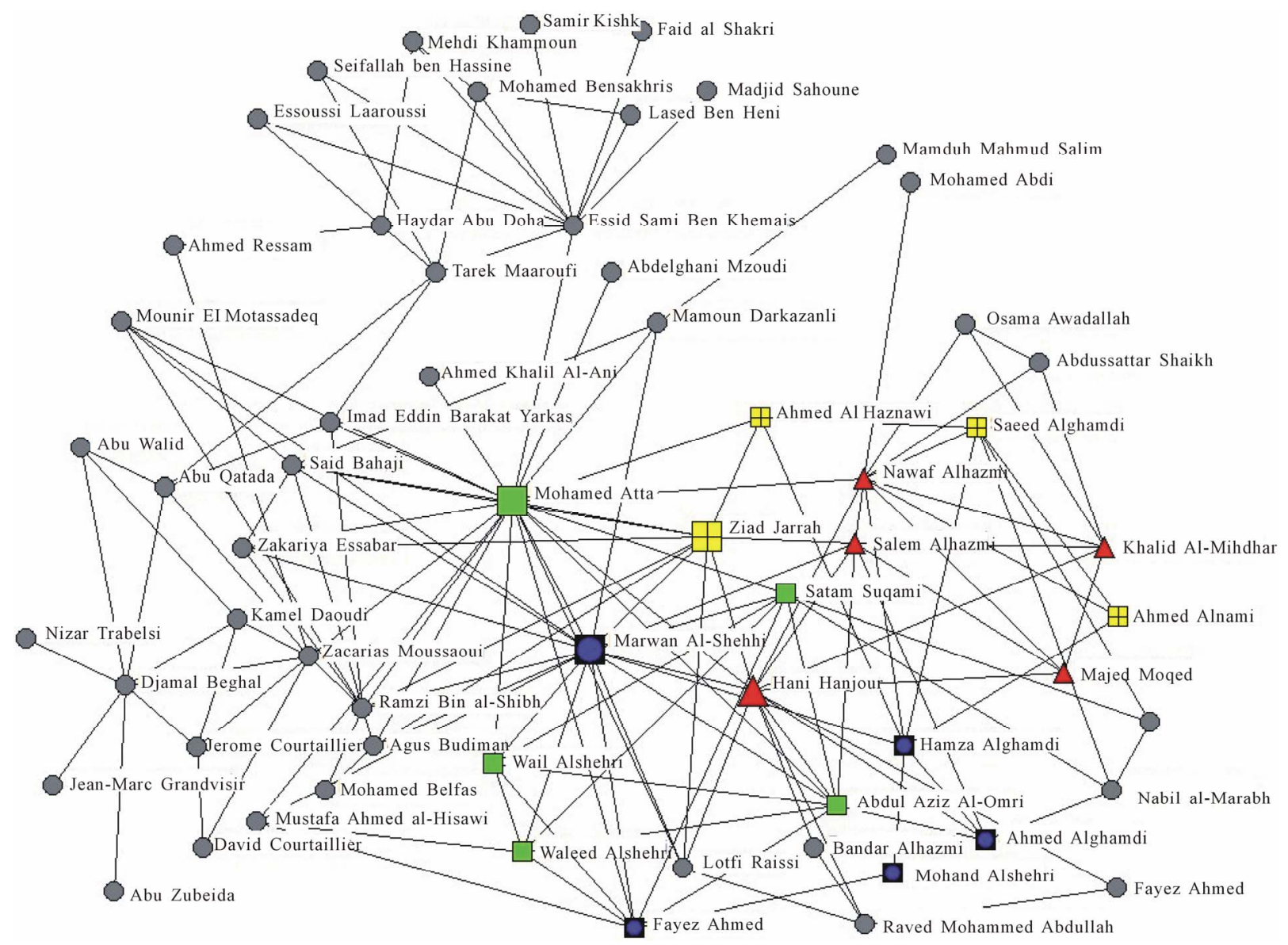

Figure 5. Valdis Krebs' (2002) data on the 9/11 hijackers. Hijackers in different flights can be distinguished by their different node shapes. Square: hijackers in Flight AA11; Up triangle: hijackers in Flight AA77; Box: hijackers in Flight UA93; Cycle in box: hijackers in Flight UA175. Larger size nodes are the pilots on each flight.

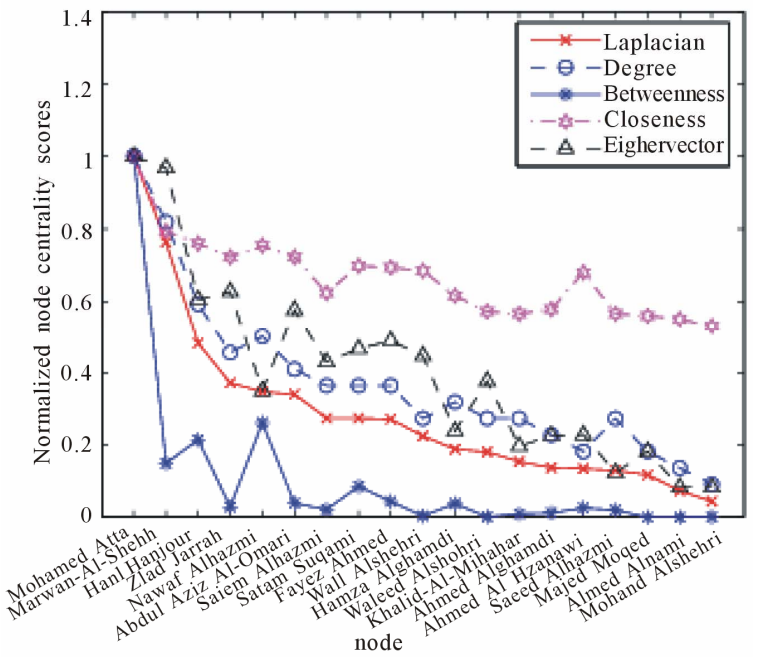

Figure 6. Normalized ranking for 19 actors in 9/11 under five centrality measurements.

from the In-School questionnaire. Each student was given a paper-and-pencil questionnaire and a copy of a roster listing every student in the school and one "sis- ter" school if it has. The question was, "List your closest (male/female) friends. List your best (male/female) friend first, then your next best friend, and so on. (Girls/Boys) may include (boys/girls) who are friends and (boy/girl) friends.” Students listed the names and corresponding numbers across a grid. Because nominations to friends in the sister school were allowed, the networks are given at the "school-pair" level. The whole data set contains 84 valued networks covering 75,871 nodes (students) in a total of 129 unique schools.

From above information, we construct the corresponding undirected networks by taking each student as a node, adding an edge between two nodes if there are nominated relationship between them. We test all five centrality methods including Laplacian method on all of these 84 networks ${ }^{1}$. To save space, here we just present

\footnotetext{
${ }^{1}$ Most of the networks are composed with a dominated connected component and several isolated vertices which have no edges with others, thus during the practical process, we actually test on the biggest connected component for each network so that we can get the two popular methods-betweenness and closeness which only work on connected graphs included to compare.
} 
Table 2. The centrality scores based on five methods for the 19 hijackers in 9/11 network; the ranks and the consensus rank.

\begin{tabular}{|c|c|c|c|c|c|c|c|c|c|c|c|}
\hline \multirow[b]{2}{*}{ Names } & \multicolumn{5}{|c|}{ Scores } & \multicolumn{5}{|c|}{ Ranks } & \multirow[b]{2}{*}{$\begin{array}{c}\text { Consensus } \\
\text { rank }\end{array}$} \\
\hline & Laplacian & Degree & Betweenness & Closeness & Eigenvector & Laplacian & Degree & Betweenness & Closeness & Eigenvector & \\
\hline $\begin{array}{l}\text { Marwan- } \\
\text { Al-Shehhi }\end{array}$ & 0.7628 & 0.8182 & 0.1495 & 0.7939 & 0.9684 & 2 & 2 & 4 & 2 & 2 & 2 \\
\hline Hani Hanjour & 0.4817 & 0.5909 & 0.2147 & 0.7591 & 0.6044 & 3 & 3 & 3 & 3 & 4 & 3 \\
\hline Ziad Jarrah & 0.3716 & 0.4545 & 0.0289 & 0.7222 & 0.6262 & 4 & 5 & 9 & 5 & 3 & 4 \\
\hline Nawaf Alhazmi & 0.3472 & 0.5000 & 0.2617 & 0.7536 & 0.3495 & 5 & 4 & 2 & 4 & 11 & 4 \\
\hline $\begin{array}{l}\text { Abdul Aziz } \\
\text { Al-Omari }\end{array}$ & 0.3399 & 0.4091 & 0.0387 & 0.7222 & 0.5752 & 6 & 6 & 7 & 5 & 5 & 6 \\
\hline Satam Suqami & 0.2738 & 0.3636 & 0.0857 & 0.6980 & 0.4660 & 7 & 7 & 5 & 7 & 7 & 7 \\
\hline Fayez Ahmed & 0.2714 & 0.3636 & 0.0438 & 0.6933 & 0.4879 & 9 & 7 & 6 & 8 & 6 & 8 \\
\hline Wail Alshehri & 0.2249 & 0.2727 & 0.0042 & 0.6842 & 0.4466 & 10 & 11 & 15 & 9 & 8 & 10 \\
\hline Hamza Alghamdi & 0.1883 & 0.3182 & 0.0376 & 0.6154 & 0.2379 & 11 & 10 & 8 & 12 & 12 & 10 \\
\hline Waleed Alshehri & 0.1809 & 0.2727 & 0.0013 & 0.5714 & 0.3762 & 12 & 11 & 16 & 14 & 10 & 12 \\
\hline $\begin{array}{c}\text { Ahmed Al } \\
\text { Haznawi }\end{array}$ & 0.1345 & 0.1818 & 0.0260 & 0.6797 & 0.2282 & 15 & 16 & 10 & 10 & 13 & 13 \\
\hline $\begin{array}{c}\text { Khalid } \\
\text { Al-Mihdhar }\end{array}$ & 0.1540 & 0.2727 & 0.0095 & 0.5652 & 0.1966 & 13 & 11 & 14 & 15 & 15 & 14 \\
\hline Ahmed Alghamdi & 0.1369 & 0.2273 & 0.0118 & 0.5778 & 0.2257 & 14 & 15 & 13 & 13 & 14 & 15 \\
\hline Saeed Alhazmi & 0.1296 & 0.2727 & 0.0198 & 0.5652 & 0.1238 & 16 & 11 & 12 & 15 & 17 & 16 \\
\hline Majed Moqed & 0.1174 & 0.1818 & 0.0000 & 0.5591 & 0.1820 & 17 & 16 & 18 & 17 & 16 & 17 \\
\hline Ahmed Alnami & 0.0733 & 0.1364 & 0.0000 & 0.5503 & 0.0825 & 18 & 18 & 18 & 18 & 18 & 18 \\
\hline Mohand Alshehri & & & & & Diviation & 13 & 52 & 125 & 30 & 73 & \\
\hline
\end{tabular}

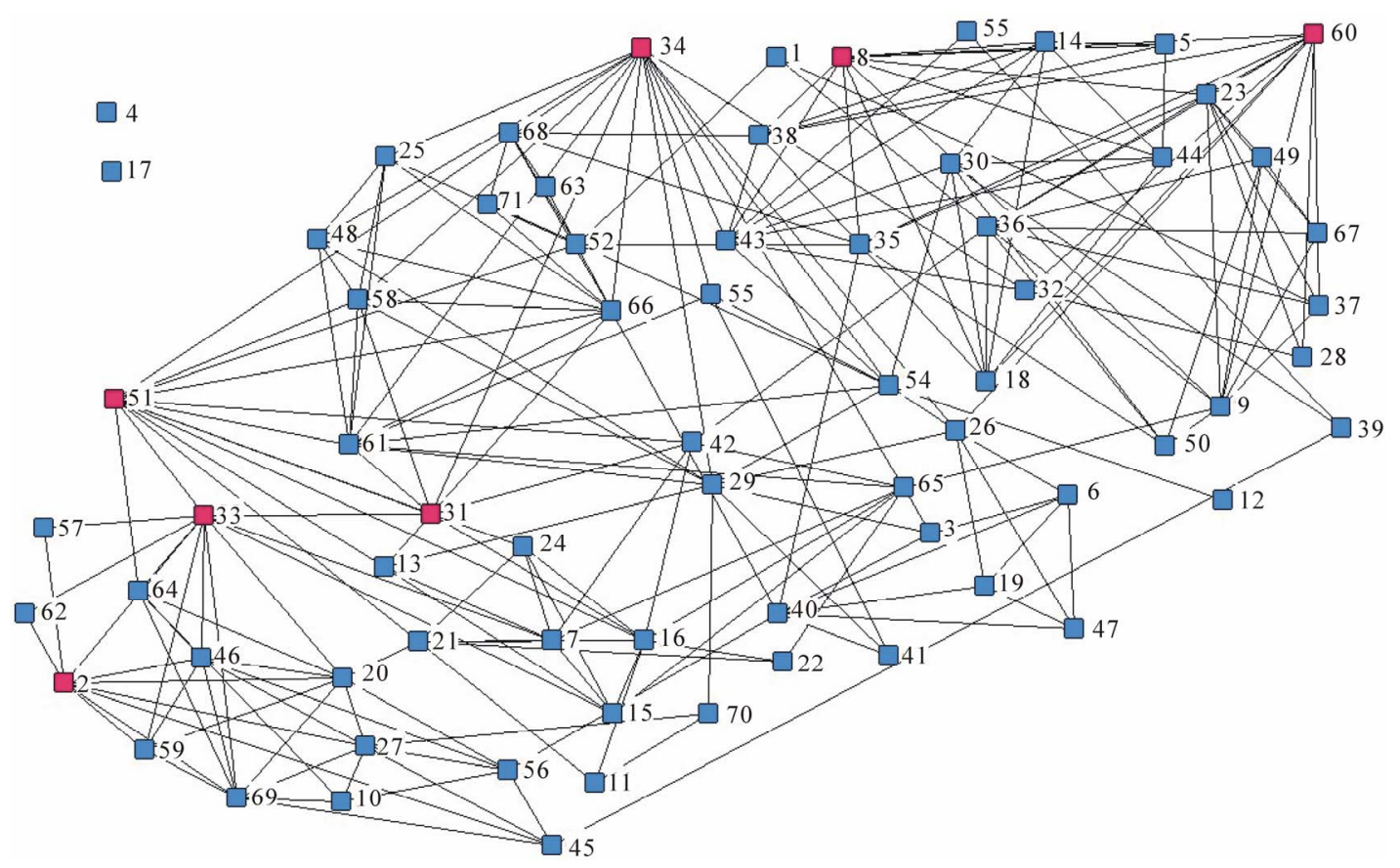

Figure 7. Net1 in the Add Health in-school friendship nomination data. 
Table 3. The centrality scores based on five methods for top 15 vertices in Net 1; their ranks and consensus rank in all 69 vertices.

\begin{tabular}{|c|c|c|c|c|c|c|c|c|c|c|c|}
\hline \multirow{2}{*}{$\begin{array}{l}\text { Node } \\
\text { number }\end{array}$} & \multicolumn{5}{|c|}{ Scores } & \multicolumn{6}{|c|}{ Ranks } \\
\hline & Laplacian & Degree & Betweenness & Closeness & Eigenvector & Laplacian & Degree & Betweenness & Closeness & Eigenvector & Consensus \\
\hline \#34 & 1 & 1 & 0.8902 & 0.9869 & 1 & 1 & 1 & 2 & 2 & 1 & 1 \\
\hline \#51 & 0.9306 & 0.9286 & 1 & 1 & 0.8879 & 2 & 2 & 1 & 1 & 3 & 2 \\
\hline \#66 & 0.7269 & 0.7857 & 0.4303 & 0.9379 & 0.8048 & 5 & 4 & 7 & 4 & 4 & 3 \\
\hline \#61 & 0.7546 & 0.7857 & 0.3080 & 0.9379 & 0.9034 & 3 & 4 & 19 & 4 & 2 & 4 \\
\hline \#33 & 0.7546 & 0.8571 & 0.7181 & 0.8118 & 0.5134 & 3 & 3 & 3 & 17 & 11 & 5 \\
\hline$\# 29$ & 0.5787 & 0.7143 & 0.3812 & 0.8728 & 0.6311 & 9 & 7 & 10 & 6 & 7 & 6 \\
\hline \#31 & 0.6204 & 0.6429 & 0.3219 & 0.9497 & 0.7546 & 7 & 10 & 18 & 3 & 6 & 7 \\
\hline$\# 54$ & 0.5278 & 0.6429 & 0.4113 & 0.8629 & 0.5562 & 13 & 10 & 8 & 9 & 10 & 8 \\
\hline$\# 43$ & 0.5185 & 0.6429 & 0.4357 & 0.8436 & 0.3713 & 14 & 10 & 6 & 14 & 13 & 9 \\
\hline$\# 60$ & 0.6759 & 0.7857 & 0.3557 & 0.8297 & 0.3157 & 6 & 4 & 13 & 16 & 20 & 10 \\
\hline$\# 16$ & 0.4815 & 0.6429 & 0.1666 & 0.8032 & 0.4308 & 19 & 10 & 24 & 18 & 12 & 11 \\
\hline \#35 & 0.4630 & 0.5714 & 0.4587 & 0.8678 & 0.2510 & 20 & 19 & 5 & 8 & 32 & 12 \\
\hline$\# 8$ & 0.6157 & 0.7143 & 0.2147 & 0.7947 & 0.2948 & 8 & 7 & 21 & 23 & 26 & 13 \\
\hline \#26 & 0.3935 & 0.5000 & 0.3599 & 0.8629 & 0.3558 & 25 & 25 & 12 & 9 & 16 & 14 \\
\hline \multirow[t]{3}{*}{$\# 36$} & 0.5509 & 0.7143 & 0.3395 & 0.7989 & 0.2422 & 10 & 7 & 15 & 21 & 35 & 15 \\
\hline & & & & & & Laplacian & Degree & Betweenness & Closeness & Eigenvector & \\
\hline & & & & & Diviation & 3314 & 4071 & 7182 & 5267 & 5854 & \\
\hline
\end{tabular}

the results for the first network Net1, which contains 71 nodes totally (a big component with 69 vertices and two isolated vertices), detailed see Figure 7. Table 3 shows all the centrality scores and ranks for 15 of these 71 vertices.

Since we are lacking of the background information of this school, it is hard for us to discuss these centrality results from known evidence. Thus in next section, we will use "deviation from consensus rank" for each centrality method to evaluate its performance. The outperformance of Laplacian method is illustrated in Table 3-the smallest deviation. For example, degree method can not distinguish the importance between node \#66 and \#61 since they have the same number of immediate neighbors, but they are distinguished by Laplacian method because Laplacian method considers the bigger environment (neighbors with 2 steps) around them.

\section{Consensus Analysis and Degree of Deviation}

Consensus analysis of outputs is a widely used method in bioinformatics for finding "consensus DNA or RNA (sub) sequence(s)" [16,17]. A similar consensus method is adapted here to evaluate the performence of various methods and, therefore, to verify the validity of Laplacian centrality method. Outputs from several standard methods are taken in consideration, and would be further compared with the consensus result. A method is regarded as more robust and has greater confidence if its output is closer (smaller deviation, or better matching) to the consensus result. Here, to save space, we demonstrate the consensus analysis only for the $9 / 11$ hijackers.

\subsection{Consensus Rank}

Different from DNA sequences whose terms are nucleotides $\{A, C, G, T\}^{2}$. The ranking of hijackers are numerically scored (natural numbers). Hence, the subjects for consensus are rather different. In this research project, we adapt the similar ideas and principles of consensus applied in bioinformation in the ranking study of social networks. The consensus ranks are calculated as follows. At first, for each terrorist, we calculate the mean of its five ranks from various methods. For example, Marwan Al-Shehhi (UA175) gets ranks $\{2,2,4,2,2\}$ from five

\footnotetext{
${ }^{2}$ Abbreviations of nucleotides: $A=$ adenine , $C=$ cytosine , $G=$ guanine,$T=$ thymine .
} 
centrality methods respectively, thus its mean of rank is 2.4 (a fractional number); secondly, we sort the mean values of these 19 hijackers from smallest to largest, the output of order is defined as the consensus rank of these 19 hijackers. Note that when ties happen (i.e., more than one hijackers have the same mean value) we follows the same criterion as used in Jemaah Islamiyah network and 9/11 network. We list the consensus rank of these 19 hijackers at the last column of Table 2.

\subsection{Deviation of Each Method from Consensus Result}

To further analyze, we use the following "deviation" score to numerically evaluate the distance from the output based on each centrality method to the consensus rank:

$$
\operatorname{deviation}(C)=\sum_{i=1}^{19}\left(\operatorname{rank}^{C}(i)-\operatorname{consensus}(i)\right)^{2}
$$

where $\operatorname{rank}^{C}(i)$ is the rank of $i$-th terrorist based on centrality measurement $C \quad(C \in$ \{Laplacian, degree, closeness, betweenness, eigenvector centrality $\}$ ), and consensus $(i)$ is the consensus rank of $i$-th terrorist.

Clearly, the smaller the deviation, the better is the output. A method with the smallest deviation is regarded as the one with "the best fit". The deviations for all centrality methods are presented at the last two rows of Table 2, which shows that Laplacian method has the smallest deviation. That is, Laplacian centrality method has the best fit to the consensus ranking results, which is a further evidence of its effectiveness and reliability to identify major players in social network.

We apply the same strategy on the Jemaah Islamiyah data set, and find that Laplacian method also has the smallest deviation, see Table 1.

We also apply the same strategy on all the 84 networks in the Add Health in-school friendship nomination data. We find that Laplacian method's deviations for all these 84 networks are always the minimum, which imply Laplacian method is the most fit centrality method for all these 84 networks. This is another strong evidence that shows the Laplacian method's advantages.

Remark The validity and robustness of Laplacian centrality measure have been illustrated on two different terrorist social network data sets and 84 networks in James Moody's Add Health in-school friendship nomination data, and is compared with other standard centrality measures. Note that this method also could be applied on large networks (e.g. number of nodes >1000) with high effectiveness (and low time-consuming) because Laplacian centrality measure has low computational complexity, and we will see in the following Section 7.

\section{Computational Complexity}

In this section, we discuss the computational complexity of Laplacian centrality method. By glancing at the definition of Laplacian centrality (Definition 2), one might initially guess that the computational complexity would be relatively high since it involves the calculation of eigenvalues. However, by applying an algebraic graph theory result (Theorem 1), we are able to design a very fast algorithm. Theorem 1 provides a structural result that graphically describes the Laplacian centrality.

For the sake of comparison, we first present the complexity of all standard methods. Let $G$ be a graph with $n$ vertices and $m$ edges. The data structure of the input graph is the adjacency list of $G$, which presents the adjacency relation of all edges of the input graph $G$.

Closeness and Betweeness Centralities. The most basic step in these two algorithms is the search for the shortest paths between every pair of vertices. Computing the shortest paths between any two vertices is the necessary step. Its fastest algorithm is Floyd-Warshall algorithm whose time complexity is $O\left(n^{3}\right)$ [18]. Hence, the total time complexity for either closeness method or betweenness method is at least $O\left(n^{3}\right)$.

Eigenvector Centrality. Given a graph, there are several approaches for estimating the eigenvector. One of the most popular approaches involves the inverses of matrices. Computational complexity is at least $O\left(n^{3}\right)$ for the computation of inverses [19].

Laplacian centrality. The degrees of all vertices and the corresponding neighborhood are estimated by scanning the adjacency list. That is, the time complexity for this step is at most $O(m)$. Then, by Theorem 1, Laplacian centralities for each one vertex $v$ needs $d(v)+2$ additive operations and 2 multiplication operations, thus the computations of centrality scores for all vertices can be finished in $O(2 m+4 n)$ units of time. Thus, the computational complexity of Laplacian method is $O(m)$.

Degree Centralities. Though degree measuring is rather intuitively heuristic and its processing is pretty straightforward, the time complexity remains $O(m)$, not smaller than that of Laplacian centrality.

To summary, we present the following table showing the time complexity of each method. We would see that Laplacian centrality offers substantial advantages to the other measures when examining large scale networks. We admit that degree method run faster than Laplacian method, but it only supplies us very local information for each vertex, which is less reliable.

\begin{tabular}{cc}
\hline laplacian & time complexity \\
\hline eigenvalue & $O(m)$ \\
betweenness & $O\left(n^{3}\right)$ \\
closeness & $O\left(n^{3}\right)$ \\
degree & $O\left(n^{3}\right)$ \\
\hline
\end{tabular}




\section{Concluding Remarks}

In this final section, we survey some properties of the new method: its balanced global/local measurement quailties, its accuracy and effectiveness, efficiency, and its future applications. The review is conducted from several very different angles: graph structure, verification of known facts from experimental testing, and the consensus for comparison.

From the graph theoretical point of view, graph centrality measurements can be roughly classified as two types: local or global. Degree centrality is a typical example of local measurement since it only considers the information of the number of vertices which are reachable from $v$ directly, while betweeness, closeness and eigenvector, are more global. Laplacian centrality reveals more connection information beyond its immediate surrounding neighborhood, thus serves as an intermediate between global and local characterizations.

Centrality measurement based on shortest path calculations (such as, betweeness, closeness, etc.) are powerful tools for the detection of bottlenecks in networks-the cut-vertices of connected graphs. Consequently, these types of vertices are scored more favorably if global connectivity is relatively low. In these lower connectivity examples, Laplacian centrality will provide a more balanced measurement, which takes both bottleneck information and local density information into account. As we discussed above, from a graph theoretical point of view different methods reveal different measurements of importance due to their different structural emphasis.

Test results from some benchmark data sets also support the conclusion that Laplacian centrality is a balanced global/local measurement. It is able to identify not only the cut point of the network of the connection, but also the leaders or key personnel in each cluster, such as the team pilot of each 911 hijacking group and the suicide bombers of bomb delivery group in the Bali nightclub attack.

Applying the new method along with other popularly used methods to two terrorist networks and all 84 friendship networks yields strong evidence that the Laplacian method provides a better performance as a network analysis tool. This is supported by two different approaches: confirmation of analyses based on known intelligence information, and consensus comparison, where the study shows that the Laplacian method has the smallest devia tion from the consensus result, providing additional evaluation of the reliability of Laplacian centrality.

In situations where scarce resources must be committed to counterterrorism programs, diversion of monitoring and surveillance from one actor to another may be a critical decision. A relatively effective and reliable method is needed for such analysis and decision making. Based on our graph theoretical analysis, testing of bench- mark data, and statistical comparisons, this newly designed method is a potentially significant candidate for a reliable tool to be used in the identification of major players in future terrorist attacks.

We have illustrated the similarities and dissimilarities with respect to the standard measures adopted in sociometry by considering some important examples of political networks. As we know, for any particular research project we will have to identify which centrality measure is most meaningful or useful. It remains to be seen, in the light of further empirical work, if and in which cases the new measure is clearly more appropriate than the others. Yet at this preliminary exposition, Laplacian centrality provides results that seem potentially compelling in certain situations where the overall degradation of the network based on vertex deletion may yield superior results. Network analysis in support of counterterrorism might well be an area where Laplacian centrality rises to such importance.

\section{Acknowledgements}

This research project is partially supported by a WV EPSCoR grant and an NSA grant H98230-12-1-0233. This work is also supported partly by Shandong Province Natural Science Foundation of China with No. ZR2010AQ018 and No. ZR2011FQ010, partly by Independent Innovation Foundation of Shandong University with No. 2010ZRJQ005, Q. Wu's work is also supported by National Natural Science Foundation of China (NO. 61202312).

\section{REFERENCES}

[1] Z. Maoz, D. Kuperman, L. Terris and I. Talmud, "Structural Equivalence and International Conflict: A Social Networks Analysis,” Journal of Conflict Resolution, Vol. 50, No. 5, 2006, pp. 664-689. doi:10.1177/0022002706291053

[2] M. Sageman, "Understanding Terror Networks," Philadelphia, University of Pennsylvania Press. 2004.

[3] J. T. Scholz, R. Berardo and B. Kile, "Do Networks Enhance Cooperation? Credibility, Search, and Collaboration," Journal of Politics, Vol. 70, No. 2, 2008, pp. 393-406.

[4] J. Fowler, B. Grofman and N. Masuoka, "Social Networks in Political Science: Hiring and Placement of Ph. Ds, 1960-2002. Political Science: 2007, pp. 727-739.

[5] S. Sreenivasan, R. Cohen, E. Lopez, Z. Toroczkai and H. E. Stanley 2007. Phys. Rev. E 75, 036105. doi:10.1103/PhysRevE.75.036105

[6] M. O. Jackson, "Presenting and Measuring networks," Chapter 2, Social and Economic Networks, Princeton University Press, Princeton, 2008.

[7] T. Opsahl, F. Agneessens and J. Skvoretz, "Node Centrality in Weighted Networks: Generalizing Degree and 
Shortest Paths,” Social Networks, Vol. 32, No. 3, 2010, pp. 245-251. doi:10.1016/j.socnet.2010.03.006

[8] F. Jordan and I. Scheuring, "Network Ecology: Topological Constraints on Ecosystems Dynamics,” Physics of Life Reviews, Vol. 1, No. 3, 2004, pp. 139-172. doi:10.1016/j.plrev.2004.08.001

[9] F. Jordan, W. C. Liu and A. J. Davis, “Topological Keystone Species: Measures of Positional Importance in Food Webs,” Oikos, Vol. 112, No. 3, 2006, pp. 535-546. doi:10.1111/j.0030-1299.2006.13724.x

[10] M. Lazic, “On the Laplacian Energy of a Graph,” Czechoslovak Mathematical Journal, Vol. 56, No. 131, 2006, pp. 1207-1213.

[11] S. Koschade, “A Social Network Analysis of Jemaah Islamiyah: The Applications to Counterterrorism and Intelligence," Studies in Conflict and Terrorism, Vol. 29, No. 6, 2006, pp. 559-575. doi:10.1080/10576100600798418

[12] V. Krebs, "Uncloaking Terrorist Networks," First Monday, Vol. 7, No. 4, 2002, 1st April.

[13] F. Chung, "Spectral Graph Theory,” American Mathe- matical Society, USA1997..

[14] J. Magouirk, S. Atran and M. Sageman, "Connecting Terrorist Networks," Studies in Conflict and Terrorism, Vol. 31, No. 1, 2008, pp. 1-16.

[15] T. H. Kean et al., “The 9/11 Commission Report,” W. W. Norton and Company, New York, 2004.

[16] M. Hamada, K. Sato and K. Asai, "Improving the Accuracy of Predicting Secondary Structure for Aligned RNA Sequences,” Nucleic Acids Research, 1-10. 2010.

[17] M. Xu, Z. Su, "Computational Prediction of cAMP Receptor Protein (CRP) Binding Sites in Cyanobacterial Genomes,” BMC Genomics, 10-23. 2009.

[18] S. Pemmaraju and S. Skiena, "All-Pairs Shortest Paths and Transitive Closure and Reduction. In: Computational Discrete Mathematics: Combinatorics and Graph Theory in Mathematica. Cambridge, England, Cambridge University Press, 2003. pp. 330-331 and 353-356.

[19] B. C. Csaji, R. M. Jungers and V. D. Blondel, "Pagerank Optimization by Edge Selection,” The Computing Research Repository abs/0911.2280. 2009. 